\title{
Storage Stabilisation of Albumin-Loaded Chitosan Nanoparticles by Lyoprotectants
}

\author{
Haliza Katas*, Zahid Hussain and Suraiya A Rahman \\ Centre for Drug Delivery Research, Faculty of Pharmacy, Universiti Kebangsaan Malaysia, Kuala Lumpur Campus, Jalan Raja \\ Muda Abdul Aziz, 50300, Kuala Lumpur, Malaysia.
}

*For correspondence: E-mail:: haliz12@hotmail.com; Tel: +6(0)392897971

Received: 10 May 2012

Revised accepted: 24 January 2013

\begin{abstract}
Purpose: To investigate the effect of lyoprotectants on the physical and storage stability of lyophilised bovine serum albumin-loaded chitosan/dextran sulphate (BSA-loaded CS/DS) nanoparticles.

Methods: BSA-loaded CS/DS nanoparticles were prepared by ionic-gelation technique. The nanoparticles were harvested by ultra-centrifugation and then various lyoprotectants at different concentrations were added to the nanoparticles prior to lyophilisation at $-40^{\circ} \mathrm{C}$ for $24 \mathrm{~h}$. Particle size and distribution as well as zeta potential of the nanoparticles were measured by dynamic light scattering method. Entrapment efficiency and BSA retained in the nanoparticles matrices were determined spectrophotometrically at $\lambda_{\max }$ of $595 \mathrm{~nm}$.

Results: The results indicate that $0.5 \% \mathrm{w} / \mathrm{v}$ trehalose was the most effective lyoprotectant and it essentially maintained the particle size of lyophilised BSA-loaded CS/DS nanoparticles which changed slightly from $188 \pm 11 \mathrm{~nm}$ to $174 \pm 14 \mathrm{~nm}$ during lyophilisation. Mannitol was also as effective as trehalose at 0.1 and $1.0 \% \mathrm{w} / \mathrm{V}$ in stabilising the nanoparticles. The particle size of lyophilized nanoparticles increased moderately from $188 \pm 11 \mathrm{~nm}$ to $234 \pm 12 \mathrm{~nm}$ and $287 \pm 18 \mathrm{~nm}$ at 0.1 and 1.0 $\%$ w/v, respectively. In contrast, the other lyoprotectants (inulin and histidine) did not show stabilizing effects. Moreover, trehalose also reduced the degree of particle aggregation from $329 \pm 16$ to $836 \pm 21$ $n m$ upon storage for $24 \mathrm{~h}$ as compared to CS/DS nanoparticles without trehalose; from $438 \pm 14$ to $1298 \pm 18(p<0.05)$. The rate of BSA leakage from the nanoparticles containing trehalose was reduced from 92 to $42 \%$ over a 11-day storage period compared with 99 to $0 \%$ for CS/DS nanoparticles without trehalose.

Conclusion: Trehalose $(0.5 \% \mathrm{w} / \mathrm{V})$ is a promising lyoprotectant for storage stabilisation of BSA-loaded CS/DS nanoparticles.
\end{abstract}

Keywords: Lyoprotectant, Chitosan, Nanoparticles, Trehalose, Bovine serum albumin, Ultracentrifugation

Tropical Journal of Pharmaceutical Research is indexed by Science Citation Index (SciSearch), Scopus, International Pharmaceutical Abstract, Chemical Abstracts, Embase, Index Copernicus, EBSCO, African Index Medicus, JournalSeek, Journal Citation Reports/Science Edition, Directory of Open Access Journals (DOAJ), African Journal Online, Bioline International, Open-J-Gate and Pharmacy Abstracts

\section{INTRODUCTION}

For the past two decades, a great deal of efforts has been focused on the fabrication of drug delivery vehicles including liposome, mixed micelles, microspheres, and polymeric nanoparticles [1,2]. Among all these, polymeric nanoparticles are more striking delivery system due to its ability to pass through the smaller capillaries and tissue gaps to arrive at target sites as well as its controlled release properties $[2,3]$. Among various materials investigated for preparing polymeric nanoparticles; chitosan (CS), a natural bio-polymer derived from chitin deacetylation $[4,5]$ has attained considerable 
attention due to its excellent biocompatibility, biodegradability and antibacterial properties [6]. Recently, polymeric nanoparticles have been prepared from chitosan and dextran sulphate (DS) with higher stability and mechanical strength as compared to chitosantripolyphosphate (CS/TPP) nanoparticles $[7,8]$. However, their clinical applications are often limited by the thermodynamic instability of their colloidal dispersion for prolonged time periods. This physical instability is mostly induced by the aggregation and precipitation of the particles in the formulation [9] and could be further aggravated by fluctuations in temperature. To alleviate these problems and to improve the storage stability of CS/DS nanoparticles, lyophilisation has been used [9]. However, the physical stresses encountered during the lyophilisation process further lead to the fusion of particles induced by dehydration-rehydration processes. To overcome this problem and to promote the physical stability of particles, carbohydrates lyoprotectants have been employed $[10,11]$. However, phase separation or crystallisation of lyoprotectants, either during the lyophilisation process or subsequent storage may adversely influence the stability of nanoparticles [12,13]. Thus, the aim of the present study was to evaluate the effects of lyophilisation process and the lyoprotective agents on the physical characteristics of CS/DS nanoparticles in order to improve their physical and storage stability.

\section{EXPERIMENTAL}

\section{Materials}

Low molecular weight chitosan (deacetylation degree (DD) 75 - $85 \%$, M. Wt $70 \mathrm{kDa}$ ), glacial acetic acid, dextran sulphate (DS), bovine serum albumin (BSA) (mol wt, 46 kDa), Bradford reagent and phosphate buffer saline (PBS) were purchased from Sigma-Aldrich, USA. Other chemicals such as mannitol and inulin were obtained from Chicory while histidine and trehalose were obtained from Sigma-Aldrich, Malaysia.

\section{Preparation of CS/DS nanoparticles}

CS/DS nanoparticles were prepared via ionicgelation method, previously developed by Calvo et al [14]. Accordingly, $100 \mathrm{mg}$ of CS was dissolved in $100 \mathrm{ml}$ of $2 \% \mathrm{v} / \mathrm{v}$ glacial acetic acid to produce $0.1 \% \mathrm{w} / \mathrm{v}$ CS solution. A concentration of $0.1 \% \mathrm{w} / \mathrm{V}$ of DS was prepared by dissolving $100 \mathrm{mg}$ of DS in $100 \mathrm{ml}$ of distilled water. CS/DS nanoparticles were simultaneously prepared by adding $4 \mathrm{ml}$ of DS solution dropwise into the $10 \mathrm{ml} 0.1 \% \mathrm{w} / \mathrm{v}$ CS solution under a constant magnetic stirring at $700 \mathrm{rpm}$ for $30 \mathrm{~min}$. Thereafter, CS/DS nanoparticles were harvested by ultra-centrifugation (25000 rpm) using Optima L-100 XP Ultracentrifuge with a rotor NV 70.1 Ti (Beckman-Coulter, USA) at $10^{\circ} \mathrm{C}$ for $15 \mathrm{~min}$.

\section{Preparation of BSA-loaded CS/DS nanoparticles}

For the association of BSA with CS/DS nanoparticles, BSA was dissolved in PBS solution to produce $0.1 \% \mathrm{w} / \mathrm{v}$ concentration. Then, BSA solution was mixed with $0.1 \% \mathrm{w} / \mathrm{v}$ CS solution and incubated for $30 \mathrm{~min}$ at room temperature. Afterward, $4 \mathrm{ml}$ of $0.1 \% \mathrm{w} / \mathrm{v}$ DS solution was added dropwise into the mixture under a constant magnetic stirring at $700 \mathrm{rpm}$ for $30 \mathrm{~min}$, to produce BSA-loaded CS/DS nanoparticles. The resultant nanoparticles were harvested by ultra-centrifugation at $25000 \mathrm{rpm}$ at $10{ }^{\circ} \mathrm{C}$ for $15 \mathrm{~min}[15]$.

\section{Lyophilisation of blank and BSA-loaded CS/DS nanoparticles}

The resultant CS/DS nanoparticles pellets were resuspended in $5 \mathrm{ml}$ of distilled water containing selected lyoprotectants (trehalose, mannitol, inulin or histidine) at different concentrations (0.1, 0.5 or $1.0 \% \mathrm{w} / \mathrm{v})$. The resulting suspensions were frozen at $-20{ }^{\circ} \mathrm{C}$ overnight prior to the lyophilisation process at a pressure of $1.3 \times 10^{6}$ atm, at $-40^{\circ} \mathrm{C}$ for $24 \mathrm{~h}$.

Determination of particle size, polydispersity index (PDI) and zeta potential

Mean particle size, PDI and zeta potential of BSA-loaded CS/DS nanoparticles with and without the addition of lyoprotectants were measured by ZS-90 Zetasizer ${ }^{\circledR}$ (Malvern Instruments, UK). Samples were either redispersed in distilled water or PBS $(\mathrm{pH} 7.4)$ prior to the measurements. All measurements were performed in triplicate at $25{ }^{\circ} \mathrm{C}$ with a detection angle of $90^{\circ}$. Results were reported as mean \pm standard deviation.

\section{Determination of entrapment efficiency (EE)}

To estimate EE, the supernatant recovered after ultra-centrifugation was decanted and the BSA content was analysed using "Bradford protein assay" as per manufacturer's instructions. The absorbance of the sample was recorded using UV/Vis-spectrophotometer (UV-1601, Shimadzu, Japan) at $\lambda_{\max }$ of $595 \mathrm{~nm}$. EE of BSA was calculated using Eq 1 [16]. 


$$
E E(\%)=\left(W_{t}-W_{f} / W_{t}\right) \times 100
$$

where, $W_{t}$ is the total initial amount of BSA and $W_{f}$ is the amount of free BSA into the supernatant recovered after ultra-centrifugation.

\section{Stability studies of BSA-loaded CS/DS nanoparticles}

Lyophilised BSA-loaded CS/DS nanoparticles were stored in desiccators for 32-days at room temperature $\left(25 \pm 2{ }^{\circ} \mathrm{C}\right)$. The sample $(100 \mathrm{mg})$ was re-hydrated and suspended in $100 \mathrm{~mL}$ of PBS ( $\mathrm{pH} 7.4,0.01 \mathrm{M})$. The percentage of BSA retained in the matrix of BSA-loaded CS/DS nanoparticles and their particle size were determined at the beginning and pre-determined time points during storage in order to assess the storage stability.

\section{Swelling analysis}

The dry samples of BSA-loaded CS/DS nanoparticles $(100 \mathrm{mg})$ with or without trehalose were immersed in $100 \mathrm{~mL}$ of PBS (pH 7.4) at room temperature $\left(25 \pm 2{ }^{\circ} \mathrm{C}\right)$ for 32 -days. The swollen samples were then collected at various day-intervals $(0,2,4,8,11,18,22,24,25$ and 32 ) by ultra-filtration. The swollen samples were then blotted with filter paper to remove excess adsorbed water from the surface of BSA-loaded CS/DS nanoparticles and weighed immediately. Swelling ratio (S) was calculated by using Eq 2 [17]

$$
S(\%)=\left(W_{s}-W_{d} / W_{d}\right) \times 100
$$

where, $W_{s}$ and $W_{d}$ are the weights of swollen and dry samples, respectively.

\section{Data analysis}

All the data were presented as mean \pm standard deviation (SD), and analysed with either paired ttest or independent t-test using SPSS 19.0. For independent t-test and paired t-test , $p<0.05$ was indicative of significant difference between the test groups.

\section{RESULTS}

\section{Effect of ultra-centrifugation on CS/DS nanoparticles}

\section{Particle size}

The mean particle size of blank CS/DS nanoparticles (without BSA) as well as the BSAloaded CS/DS nanoparticles was significantly influenced $(p<0.05$, unpaired t-test) by the process of ultra-centrifugation. Resulting data suggested that the mean particle size of blank CS/DS nanoparticles decreased from $303 \pm 16$ $\mathrm{nm}$ to $193 \pm 8 \mathrm{~nm}$, after ultra-centrifugation. Similarly, the particle size of BSA-loaded CS/DS nanoparticles was also reduced from $301 \pm 14$ $\mathrm{nm}$ to $206 \pm 6 \mathrm{~nm}$.

\section{Zeta potential and polydispersity index (PDI)}

The data obtained depict that the zeta potential of blank CS/DS nanoparticles was significantly decreased $(p<0.05)$ from $+67 \pm 4 \mathrm{mV}$ to $+31 \pm$ $0.6 \mathrm{mV}$ after ultra-centrifugation. The average surface charge of BSA-loaded CS/DS nanoparticles was also remarkably decreased $(p<0.05)$ from $+69 \pm 4 \mathrm{mV}$ to $+25 \pm 3 \mathrm{mV}$ Furthermore, the mean value of PDI for the blank and BSA-loaded CS/DS nanoparticles was considerably reduced from $0.318 \pm 0.001$ and $0.335 \pm 0.02$ to $0.149 \pm 0.006$ and $0.162 \pm 0.006$ respectively, after ultra-centrifugation which indicate that their particle size was narrowly distributed.

\section{Effects of types and concentrations of lyoprotectants}

Fig 1 (A) represents the various modulations of the particle size of BSA-loaded CS/DS nanoparticles containing different types and concentrations of lyoprotectants before and after lyophilisation process. Before lyophilization, the blank CS/DS nanoparticles without lyoprotectant (control) showed significant increase $(p<0.05)$ in the particle size from $193 \pm 8$ to $351 \pm 13 \mathrm{~nm}$ after lyophilisation. Similar to that, the zeta potential of the control had shown a considerable decrease $(p<0.05)$ from $+31 \pm 0.6$ to $+23 \pm 3.5$ $\mathrm{mV}$ after lyophilisation process as depicted by Fig 1(B). The PDI value of the control group showed significant increase by 3.2-fold to $0.49 \pm$ 0.01. On the other hand, the BSA-loaded CS/DS nanoparticles had also shown a significant $(p<$ $0.05)$ variation in the particle size from $206 \pm 6$ to $363 \pm 18 \mathrm{~nm}$ and in the zeta potential from $+25 \pm$ 3 to $19 \pm 2 \mathrm{mV}$ after lyophilization. To circumvent this problem, different types of lyoprotectants were investigated as shown in Fig 1. It was found that the particle size of CS/DS nanoparticles containing $0.5 \% \mathrm{w} / \mathrm{v}$ trehalose remained essentially unchanged after lyophilisation. Inaddition, the mannitol was as effective as trehalose in maintaining the particle size of CS/DS nanoparticles at 0.1 and $1.0 \% \mathrm{w} / \mathrm{v}(p>$ $0.05)$. However, at a concentration of $0.5 \% \mathrm{w} / \mathrm{v}$, mannitol was less effective than trehalose. In contrast, other lyoprotectants (inulin and histidine) were clearly less effective in this regard, as shown in Fig. 1A. Furthermore, the 
surface charge of CS/DS nanoparticles was least affected by lyophilization when trehalose was used as stabilizer compared to the other lyoprotectants as shown in Fig 1(B). The results suggested that trehalose was very effective at 0.1 and $0.5 \% \mathrm{w} / \mathrm{v}$ concentration at maintaining the zeta potential of CS/DS nanoparticles. On the other hand, the other lyoprotectants did not prevent the adverse effects of lyophilization (Fig $1 \mathrm{~B})$. Thus, the ,trehalose $(0.5 \% \mathrm{w} / \mathrm{v})$ was the most effective agent in preserving the physical characteristics of CS/DS nanoparticles, and therefore, was chosen for further experiments.

$\begin{array}{ll}\text { 영 Before freeze-drying } & \text { m Lyophillized without lyoprotectant } \\ \text { 圈 With trehalose } & \text { With manitol } \\ \text { g With inuline } & \text { W With histidine }\end{array}$

Fig 1: Effects of different types and concentrations of lyoprotectants on (A) particle size and (B) zeta potential of CS/DS nanoparticles. ${ }^{*} \mathrm{CS} / \mathrm{DS}$ nanoparticles were rehydrated and suspended in distilled water, $\mathrm{n}=3$.

\section{Storage stability of BSA-loaded CS/DS nanoparticles}

It was addressed by the previous experiments that trehalose was the most efficient to stabilize lyophilized CS/DS nanoparticles. Taken together, the stabilization effect of trehalose on CS/DS nanoparticles was also accessed upon storage. Three parameters were measured: 1) mean particle size, 2) zeta potential 3) percent of BSA retained in the matrices of CS/DS nanoparticles. For this experiment, the EE of BSA-loaded CS/DS nanoparticles containing $0.5 \% \mathrm{w} / \mathrm{v}$ trehalose was $92 \%$ prior to the lyophilisation process. On the other hand, EE for BSA-loaded CS/DS nanoparticles without the addition of trehalose was $98 \%$ and was used as a control.

\section{Particle size and zeta potential of CS/DS nanoparticles}

Results highlighted in Fig 2 suggested that trehalose could protect the nanoparticles from forming aggregates induced by lyophilisation process. The mean particle size of control sample (lyophilised nanoparticles without trehalose $0.5 \% \mathrm{w} / \mathrm{v}$ ) was significantly increased from $438 \pm 14$ to $1298 \pm 18(p<0.05$, paired ttest) from day-0 to day-32, which indicated extensive particle aggregation as expressed by Fig 2. In contrast to that, the average particle size of lyophilised BSA-loaded CS/DS nanoparticles containing trehalose $(0.5 \% \mathrm{w} / \mathrm{v})$ increased gradually from $329 \pm 5$ to $836 \pm 6(p<$ 0.05 , paired t-test) from day-0 to day-32.

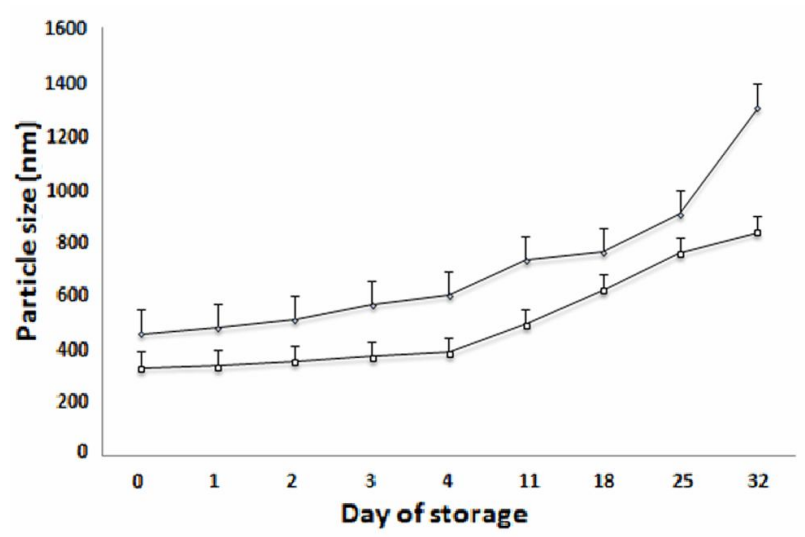

Fig 2: Effect of storage of BSA-loaded CS/DS nanoparticles (CS $0.1 \% \mathrm{w} / \mathrm{v}$, DS $0.1 \% \mathrm{w} / \mathrm{v}$, BSA $0.1 \% \mathrm{w} / \mathrm{v}$ ) with (口) and without $(\diamond)$ trehalose $0.5 \% \mathrm{w} / \mathrm{v}$ on particle size. The nanoparticles were re-hydrated and suspended in PBS $(\mathrm{pH} 7.4, \mathrm{n}=3)$

Moreover, Table 1 shows the modulation of zeta potential of BSA-loaded CS/DS nanoparticles with and without trehalose $0.5 \% \mathrm{w} / \mathrm{v}$. The zeta potential of nanoparticles with trehalose $0.5 \%$ $\mathrm{w} / \mathrm{v}$ was more negatively charged than CS/DS nanoparticles without trehalose -during storage.

\section{BSA retention in CS/DS nanoparticles}

The results presented in Fig 3 demonstrate that the leakage of BSA from BSA-loaded CS/DS nanoparticles into the suspending medium progressed slowly in the presence of trehalose 
Table 1: Effect of trehalose $(0.5 \% \mathrm{w} / \mathrm{v})$ on zeta potential and PDI of BSA-loaded CS/DS nanoparticles upon storage (mean $\pm S D, n=3$ ).

\begin{tabular}{|c|c|c|c|c|}
\hline \multirow{2}{*}{$\begin{array}{l}\text { Storage } \\
\text { Period } \\
\text { (day) }\end{array}$} & \multicolumn{2}{|c|}{$\begin{array}{l}\text { BSA-loaded CS/DS NPs without trehalose } \\
\qquad(0.5 \% \mathrm{w} / \mathrm{v})\end{array}$} & \multicolumn{2}{|c|}{$\begin{array}{l}\text { BSA-loaded CS/DS NPs with trehalose }(0.5 \% \\
\text { w/v) }\end{array}$} \\
\hline & $P D I$ & Surface charge (mV) & PDI & $\begin{array}{c}\text { Surface charge } \\
(\mathrm{mV})\end{array}$ \\
\hline 0 & $0.50 \pm 0.06$ & $-8.00 \pm 0.30$ & $0.34 \pm 0.01$ & $-15.00 \pm 0.82$ \\
\hline 1 & $0.61 \pm 0.01$ & $-7.00 \pm 0.51$ & $0.37 \pm 0.01$ & $-11.00 \pm 0.15$ \\
\hline 2 & $0.58 \pm 0.12$ & $-8.00 \pm 0.28$ & $0.54 \pm 0.05$ & $-11.00 \pm 0.91$ \\
\hline 3 & $0.59 \pm 0.01$ & $-12.00 \pm 1.84$ & $0.61 \pm 0.02$ & $-16.00 \pm 0.55$ \\
\hline 4 & $0.58 \pm 0.12$ & $-10.00 \pm 1.13$ & $0.45 \pm 0.01$ & $-13.00 \pm 0.41$ \\
\hline 11 & $0.49 \pm 0.004$ & $-9.00 \pm 0.02$ & $0.56 \pm 0.02$ & $-12.00 \pm 0.46$ \\
\hline 18 & $0.71 \pm 0.11$ & $-7.00 \pm 1.04$ & $0.39 \pm 0.01$ & $-16.00 \pm 0.15$ \\
\hline $\begin{array}{l}25 \\
32\end{array}$ & $\begin{array}{l}0.59 \pm 0.01 \\
0.60 \pm 0.004\end{array}$ & $\begin{array}{l}-7.00 \pm 0.55 \\
-4.00 \pm 0.77\end{array}$ & $\begin{array}{l}0.49 \pm 0.01 \\
0.23 \pm 0.00\end{array}$ & $\begin{array}{l}-12.00 \pm 1.13 \\
-9.00 \pm 0.80\end{array}$ \\
\hline
\end{tabular}

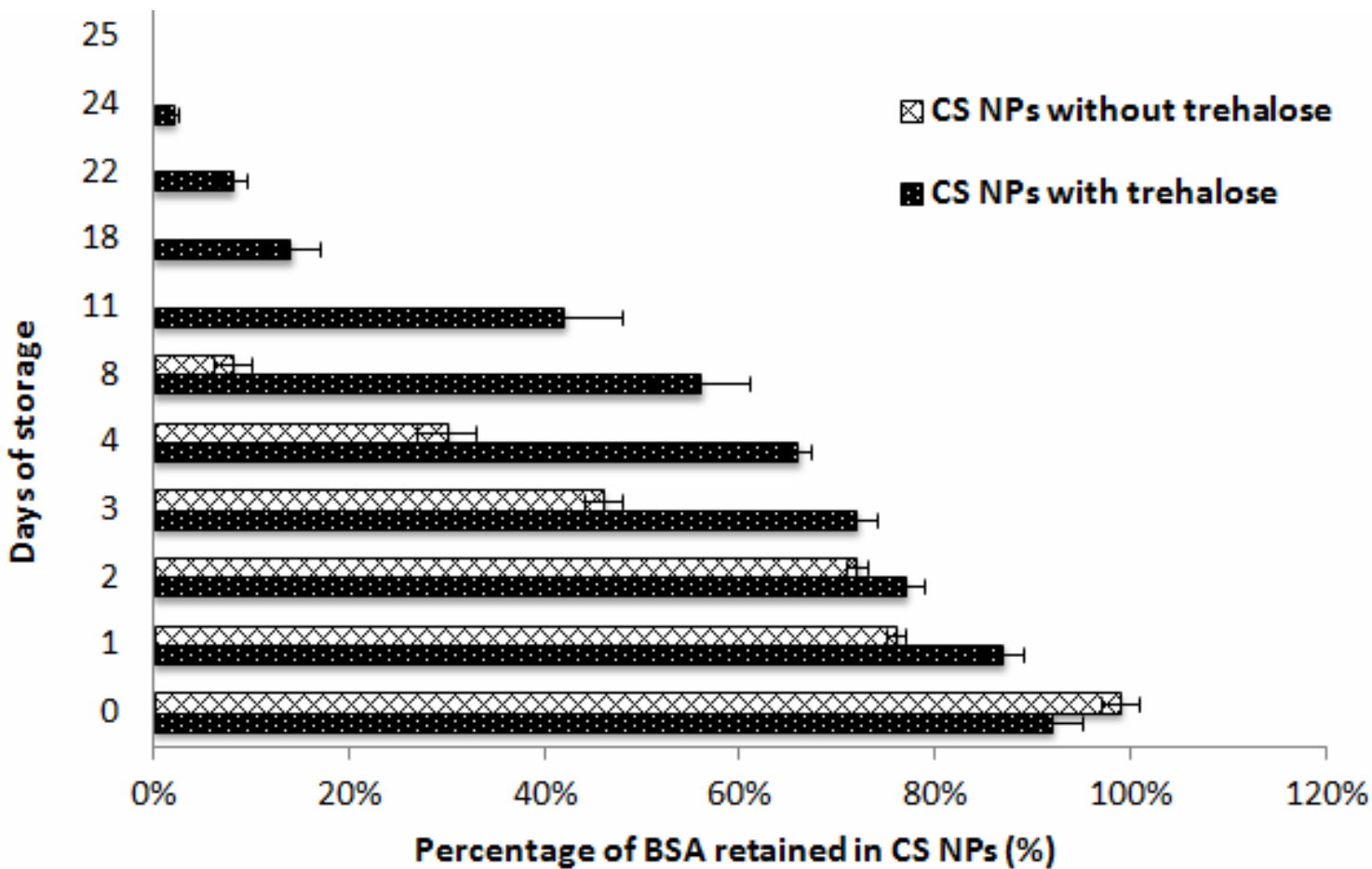

Fig 3: Effect of storage of BSA-loaded CS/DS nanoparticles (CS $0.1 \% \mathrm{w} / \mathrm{v}$, DS $0.1 \% \mathrm{w} / \mathrm{v}$, BSA $0.1 \% \mathrm{w} / \mathrm{v}$ ) with or without trehalose $0.5 \% \mathrm{w} / \mathrm{v}$ on the percent of BSA retained in the matrix of CS/DS nanoparticles. ${ }^{*} \mathrm{CS} / \mathrm{DS}$ nanoparticles were rehydrated and suspended in PBS $(\mathrm{pH} 7.4, \mathrm{n}=3)$

$(0.5 \% \mathrm{w} / \mathrm{v})$. The resulting data revealed that the percent of BSA retained was reduced from $92 \%$ to $42 \%$ from Day-0 to 11 . The percent of drug retained showed an abrupt fall to $14 \%$ at Day-18. Furthermore, the percent of BSA retained dropped to zero at day-25 of storage. In contrast to that, BSA rapidly leaked from the CS/DS nanoparticles without trehalose from $99 \%$ to $8 \%$ from day-0 to day-8. Thus, the CS/DS nanoparticles without trehalose were observed to be completely lost their active content at day-11 as shown in Fig 3 .

\section{Swelling studies}

In order to investigate the effects of storage on the swelling characteristics of BSA-loaded CS/DS nanoparticles, a series of experiments were carried out from day-0 to 32 . Fig 4 reveals that the swelling ratios of BSA-loaded CS/DS nanoparticles without trehalose was observed to be significantly increased $(p<$ 0.05 , student t-test) from $26 \%$ to $81 \%$ from day-2 to 32. In addition to that, the swelling ratio of CS/DS nanoparticles containing trehalose $0.5 \% \mathrm{w} / \mathrm{v}$ was significantly lower $(p<$ 0.05 , student t-test) than the ones without trehalose as shown by Fig 4 . 


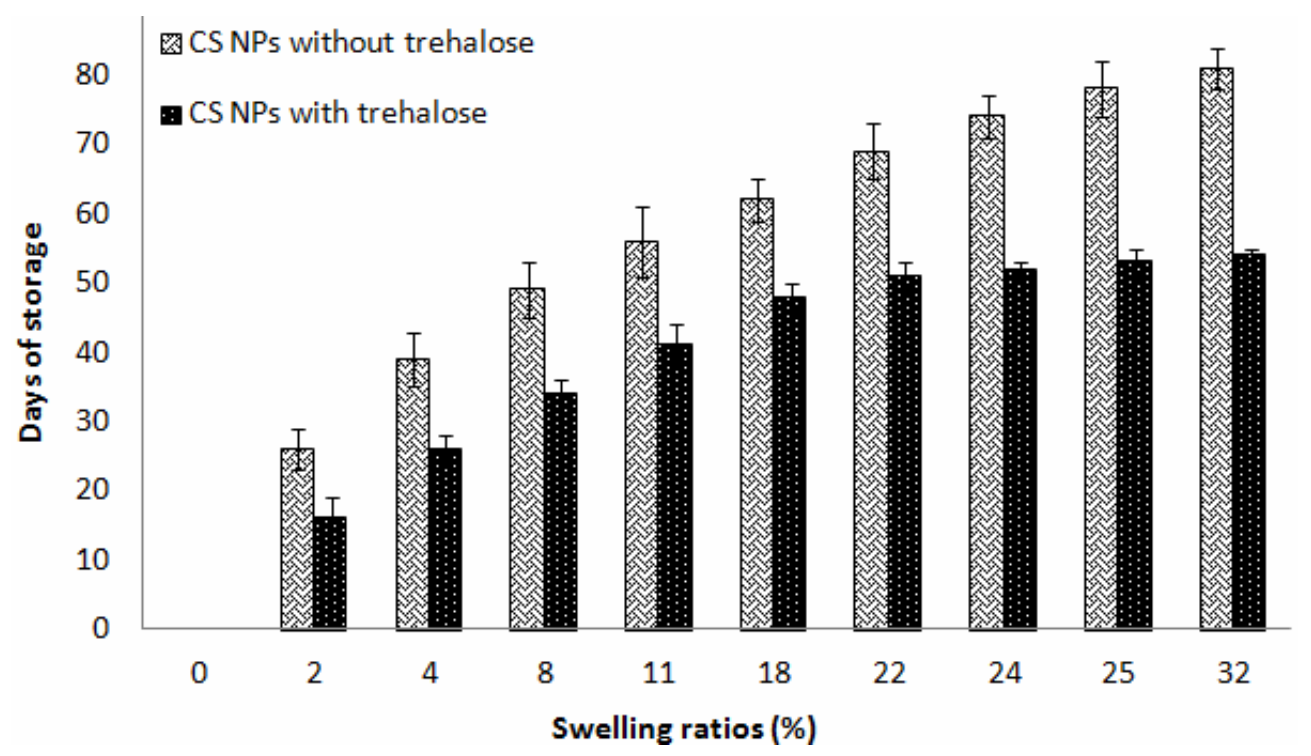

Fig 4: Effect of trehalose $0.5 \% w / v$ on the swelling ratio of BSA-loaded CS/DS nanoparticles upon storage. ${ }^{*} \mathrm{CS} / \mathrm{DS}$ nanoparticles were rehydrated and suspended in PBS $(\mathrm{pH} 7.4, \mathrm{n}=3)$

\section{DISCUSSION}

The data obtained from the study suggested that ultra-centrifugation process may adversely affects the physical characteristics of CS/DS nanoparticles, which were also corroborated with the previous findings reported by Katas et al [18]. This phenomenon could be attributed to the reason that smaller particles may adsorb on the surface of larger particles via partial physical interactions to form agglomerates. However, during ultra-centrifugation process, the surface adsorbed particles could be washed away from the larger particles due to high centrifugation speed [18]. Similarly, the average zeta potential and PDI of nanoparticles was also observed to be affected by centrifugation process.

Fig 1 illustrate that trehalose was more efficient in preventing the drastic modification of the physical characteristics of lyophilised CS/DS nanoparticles. The particle size of lyophilised nanoparticles with trehalose was the least affected by the process of lyophilisation. On the other hand, other lyoprotectants such as mannitol, inulin and histidine were less effective in preventing particle aggregation induced by lyophilisation process. Moreover, the zeta potential of CS/DS nanoparticles containing inulin or histidine was also observed to be reduced significantly $(p<0.05$, paired t-test). The low surface charge value of CS/DS nanoparticles in the presence of inulin or histidine contributed to the relatively lesser repulsive forces among individual particles and therefore, they tend to form aggregates. This was expected to be due to the crystallisation of both inulin and histidine during lyophilisation which was also in accordance with the theories proposed by Mohammed et al and Andre et al $[12,19]$. They reported that inulin exists in two crystalline forms which are semi-hydrated and hydrated and thus, the particles tend to form agglomerates.

Moreover, PDI is crucially important to be evaluated as it indicates the conservation of particle size distribution. Results depicted that the PDI values of CS/DS nanoparticles with the addition of inulin and histidine were significantly increased ( $p<0.05$, paired t-test) compared to the controls (nanoparticles without lyoprotectant and before lyophilisation process). Based on these results, it was elucidated that all other lyoprotectants except trehalose were shown to be less effective in protecting CS/DS nanoparticles from forming aggregates caused by the dehydration and other physical stresses during lyophilisation process. These results were also corroborated with other findings reported in previous studies [11-13].

A significant increase in the particle size of nanoparticles was also observed with all other concentrations of trehalose $(0.1$ and $1.0 \% \mathrm{w} / \mathrm{v})$. This finding therefore suggested that the other concentrations could not sufficiently protect the lyophilised nanoparticles as achieved by \%w/v trehalose 0.5 . This was thought to be caused by the destabilisation of the nanoparticles due to the fact that trehalose might have reached its limit of destabilisation [9-11].

Trehalose $0.5 \% \mathrm{w} / \mathrm{v}$ was found to be able to retain the particle size of lyophilised BSA-loaded CS/DS nanoparticles up to day-4 of storage (Fig 
2). A significant increase in their particle size was only observed from day-11 of storage. The pretreatment of CS/DS nanoparticles with trehalose would not only preserve the particle size efficiently, but also minimise the drug leakage from nanoparticles matrices $[15,19]$. The desorption of loosely bound BSA molecules from the surface of the CS/DS nanoparticles was reported to be responsible for the higher rate and extent of BSA leakage from nanoparticles matrices without trehalose when they were suspended in a medium. In contrast to that, a comparatively lower rate and extent of BSA leakage from the BSA-loaded CS/DS nanoparticles in the presence of trehalose could be due to the formation of an extra protective barrier on the nanoparticles surfaces which could slow down the rapid leakage of BSA as shown in Fig 3.

CS/DS nanoparticles without trehalose had a higher swelling percentage as compared to the nanoparticles with trehalose $0.5 \% \mathrm{w} / \mathrm{v}$. It was expected as the CS/DS nanoparticles without trehalose were directly exposed to the external environment (PBS, pH 7.4), which caused the $\mathrm{NH}_{3}{ }^{+}$groups on the contour of CS to change into neutral $-\mathrm{NH}_{2}$ group. As a result, the electrostatic interactions between CS and DS became extensively weakened which favours the swelling of CS/DS nanoparticles. However, the addition of trehalose into the formulation had allowed an extra lyoprotective layer to be formed on the surface of nanoparticles and protect them from external environment (PBS, pH 7.4). This could therefore cause the cross-linking efficiency to be remained stable which subsequently inhibited the particle from swelling.

\section{CONCLUSION}

In this present research, the successful loading of BSA into the CS/DS nanoparticles was achieved via ionic-gelation method. It was demonstrated that the physical characteristics (particle size, PDI and zeta potential) of BSAloaded CS/DS nanoparticles were significantly influenced by the process of lyophilisation. To circumvent this physical instability induced by lyophilisation, various lyoprotective agents have been investigated. It was observed that $0.5 \%$ $w / v$ trehalose was more effective to physically stabilise the lyophilised BSA-loaded CS/DS nanoparticles. Data obtained also suggested that $0.5 \% \mathrm{w} / \mathrm{v}$ trehalose could be used to reduce the rate and extent of BSA leakage upon storage. These findings therefore suggest that trehalose could be used to provide physical protection during lyophilisation and to improve the storage stability of BSA-loaded CS/DS nanoparticles.

\section{ACKNOWLEDGMENT}

Authors gratefully acknowledge "Dana Lonjakan Penerbitan" of Universiti Kebangsaan Malaysia (UKM-DLP-2011-001) for funding this research project.

\section{REFERENCES}

1. Du YZ, Wang L, Yuan $H$, Wei XH, Hu FQ. Preparation and characteristics of linoleic acid-grafted chitosan oligosaccharide micelles as a carrier for doxorubicin. Colloid Surface B. 2009; 69: 257-263.

2. Jung $T, K a m m$, Breitenbach A, Kaiserling E, Xiao JX, Kissel T. Biodegradable nanoparticles for oral delivery of peptides: is there a role for polymers to affect mucosal uptake? Eur J Pharm. Biopharm. 2000; 50: 147-160.

3. Liu ZH, Jiao YP, Wang YF, Zhou CR, Zhang ZY. Selfassembly of protein with polymer. Adv Drug Deliver Rev. 2008; 60: 1650-1662.

4. Cooney MJ, Petermann J, Lau C, Minteer SD. Characterization and evaluation of hydrophobically modified chitosan scaffolds: Towards design of enzyme immobilized flow-through electrodes. Carbohyd Polym. 2009; 75: 428-435.

5. Peesan M. Supaphol $P$, Rujiravanit $R$. Preparation and characterization of hexanoyl chitosan/polylactide blend films. Carbohyd Polym. 2005; 60: 343-350.

6. No HK, Kim SH, Lee SH, Park NY, Prinyawiwatkul W. Stability and antibacterial activity of chitosan solutions affected by storage temperature and time. Carbohyd Polym. 2006; 65: 174-178.

7. Calvo P, Remunan C, Vila JJL, Alonso MJ. Novel hydrophilic chitosan-polyethylene oxide nanoparticles as protein carriers. J Appl Polym Sci. 1997; 63: 125-132.

8. Chen Y, Vellore J, Mohanraj, Wang F, Benson HAE. Designing Chitosan-Dextran Sulphate Nanoparticles Using Charge Ratios. AAPS Pharm. Sci. Tech. 2006; 8: 131-139.

9. Musumeci $T$, Vicari $L$, Ventura CA, Gulisano $M$, Pignatello R, Puglisi G. Lyoprotected nanosphere formulations for paclitaxel controlled delivery. $J$ Nanosci Nanotech. 2006; 6: 457-503.

10. Abdelwahed W, Degobert G, Fessi H. A pilot study of freeze drying of poly(epsilon-carprolactone) nanocapsules stabilized by poly(vinyl alcohol): formulation and process optimization. Int $J$ Pharm. 2006; 309: 178-188.

11. Zhang $L$, Liu $L$, Qian $Y$, Chen $Y$. The effects of cryoprotectants on the freeze-drying of ibuprofenloaded solid lipid microparticles (SLM). Eur J Pharm Biopharm. 2008; 69: 750-759

12. Mohammed $A R$, Allan GA, Coombes and Yvonne Perrie. Amino acids for liposomal delivery systems. Eur J Pharm Sci. 2007; 30(5): 406-413.

13. Sundaramurthi $P$, Patapoff TW, Suryanarayanan $R$. Crystallization of trehalose in frozen solutions and its phase behavior during drying. Pharmaceut Res. 2010; 27(11): 2374-2383

14. Calvo $P$, Remuñan-López C, Vila-Jato JL, Alonso MJ. Chitosan and chitosan/ethylene oxide-propylene oxide block copolymer nanoparticles as novel carriers for proteins and vaccines. Pharmaceut Res. 1997; 14: 1431-1436

15. Rahimnejad M, Jahanshahi M, Najafpour GD. Production of biological nanoparticles from bovine serum albumin for drug delivery. Afr J Biotech. 2006; 5(20): 1918-1923

16. Papadimitriou S, Bikiaris D, Avgoustakis K. Chitosan nanoparticles loaded with dorzolamide and pramipexole. Carbohyd Polym. 2008; 73: 44-54. 
17. Dai ZZ, Yin JB, Yan SF, Cao $T$, Ma J, Chen XS. Polyelectrolyte complexes based on chitosan and poly (L-glutamic acid). Polym Int. 2007; 56: 1122 1127.

18. Katas H, Chen S, Osamuyimen AA, Cevher E, Oya Alpar $H$. Effect of preparative variables on small interfering loaded Poly(D,L-Lactide-Co-Glycolide)- chitosan submicron particles prepared by emulsification diffusion method. J Microencapsul. 2008: 25(8): 541-548.

19. D'Andrea $G$, Salucci $M L$, Avigliano $L$. Effect of lyoprotectants on ascorbate oxidase activity after freeze-drying and storage. Process Biochem. 1996; 31: 173-178. 